\title{
Chronic Kidney Disease: A Place for Primary Care and Nephrology to Meet
}

\author{
Deidra C. Crews, MD, SCM $1,2,3,4$
}

'Division of Nephrology, Department of Medicine, Johns Hopkins University School of Medicine, Baltimore, MD, USA; ${ }^{2}$ Welch Center for Prevention, Epidemiology and Clinical Research, Johns Hopkins Medical Institutions, Baltimore, MD, USA; ${ }^{3}$ Center on Aging and Health, Johns Hopkins Medical Institutions, Baltimore, MD, USA; ${ }^{4}$ Hopkins Center to Eliminate Cardiovascular Health Disparities, Johns Hopkins Medical Institutions, Baltimore, MD, USA.

J Gen Intern Med 31(1):5-6

DOI: $10.1007 / \mathrm{s} 11606-015-3506-0$

(c) Society of General Internal Medicine 2015

$I^{\mathrm{n}}$ $\mathrm{n}$ this issue of JGIM, Ricardo et al. report findings from the Chronic Renal Insufficiency Cohort (CRIC) study in their manuscript titled 'Influence of Nephrologist Care on Management and Outcomes in Adults with Chronic Kidney Disease'. ${ }^{1}$ The authors characterize patterns of nephrology care and examine the association between receipt of nephrology care and guideline-concordant management of chronic kidney disease (CKD) complications and clinical outcomes. The article highlights a number of key issues, including disparities in self-reported receipt of nephrology care. When clinical outcomes were examined, Ricardo et al. found that prior nephrology contact was associated with some guidelineconcordant treatments of CKD complications among a subset of patients with CKD stage 4; however, in this population recruited from general medicine and specialty clinics in the U.S., there was no statistically significant association between prior nephrology contact and CKD progression, incident cardiovascular disease (CVD) or death.

As is the case for most chronic conditions, primary care providers (PCPs) are generally the first to detect that a patient has CKD. Thereafter, a PCP may or may not refer their patient to be seen by a nephrologist. Current guidelines recommend that patients with an estimated glomerular filtration rate (eGFR) $<30 \mathrm{ml} / \mathrm{min} / 1.73 \mathrm{~m}^{2}$ and/or severely increased albuminuria (i.e. urinary albumin-to-creatinine ratio $>300 \mathrm{mg} / \mathrm{g}$ ) be referred to a nephrologist. ${ }^{2}$ Other indications for referral include rapid eGFR decline ( $>5 \mathrm{ml} / \mathrm{min} / 1.73 \mathrm{~m}^{2}$ per year), hematuria and uncontrolled complications of CKD (ie. hypertension requiring four or more antihypertensive agents, anemia and/or electrolyte abnormalities). ${ }^{2}$ In addition, PCPs should consider referring their CKD patients to nephrology if they come from demographic groups known to experience more rapid disease progression, including young adults and racial/ethnic minorities.

Ricardo et al. found that $67 \%$ of CRIC participants, who all had CKD stages 1 to 4 at baseline, reported having seen a

Published online September 10, 2015 nephrologist prior to study enrollment. This is in stark contrast to the Medicare population aged 65 and older, where it is estimated that less than a third of patients with a CKD claim in 2011 were seen by a nephrologist over the subsequent year. ${ }^{3}$ The much greater prevalence of nephrology care among CRIC participants was likely due to many having been referred to the study by their nephrologist. In addition, most CRIC participants were receiving care at clinics closely affiliated with academic medical centers, and based in urban settings, which would be expected to have considerable availability of subspecialty care. On the contrary, in rural communities, CKD frequently goes undocumented in primary care, and co-management with nephrology is uncommon. ${ }^{4}$

Based upon a multivariable analysis, Ricardo et al. identified multiple sociodemographic factors independently associated with lesser odds of receiving nephrology care, including older age, female gender, Hispanic ethnicity, low socioeconomic status (as measured by educational attainment and insurance status), diabetes and higher body mass index. These findings may have been due to patient factors, including preferences regarding the number of providers involved in their care and lack of adherence to scheduled appointments (which was not captured in the CRIC study). Possible contributing provider factors worth consideration include PCPs biases regarding which patients would be able to understand and accept a diagnosis of CKD. A prior qualitative study found that PCPs felt CKD was quite difficult to explain to patients with low literacy, and they also worried about emotionally overwhelming their patients with a diagnosis of CKD. ${ }^{5}$ These concerns could contribute to lower rates of nephrology referral for certain patient groups.

While PCPs and nephrologists generally desire to engage in collaborative care for CKD patients, ${ }^{6}$ there is uncertainty among both attending generalists ${ }^{7}$ and internal medicine residents ${ }^{8}$ about which clinical findings should trigger a referral-which is an opportunity for broader dissemination of CKD guidelines. Furthermore, PCPs may question the value of subspecialist care for a condition closely linked to two common chronic conditions primarily managed by PCPs - diabetes and hypertension. In fact, some PCPs do not view $\mathrm{CKD}$ as a separate chronic condition requiring additional management. ${ }^{4}$ So, what can the nephrologist add? 
Beyond treating complications of $\mathrm{CKD}$, as suggested by the CRIC study findings that CKD stage 4 patients under the care of a nephrologist were more likely to achieve certain clinical targets (e.g. treatment-associated serum phosphate control), nephrologists also contribute to the care of CKD patients in ways which are difficult to capture in cohort studies such as CRIC. For example, nephrologists are better positioned than PCPs to determine the etiology of CKD (often aided by a kidney biopsy) and establish specific treatment plans (ie. immunosuppression). This is a particularly important consideration in the care of diabetic patients with CKD, many of whom may have CKD due to other causes which will not be addressed by glycemic control. Nephrologists may also educate patients about their disease, including discussions about avoiding potentially harmful substances, and provide expectant guidance informed by their assessment of trajectories of kidney function decline and albuminuria. Furthermore, the potential for lifestyle modifications spurred by patients being told they have CKD by a nephrologist should not be discounted. As emerging evidence reveals that dietary ${ }^{9}$ and other lifestyle factors ${ }^{10}$ may influence outcomes in CKD, patients' access to nephrology care becomes all the more vital to safely and effectively empowering them to alter their own disease course. For the minority of CKD patients who progress to requiring renal replacement therapy (most die prior to reaching ESRD), nephrologists may also be better suited to detail treatment options and prepare them for timely referral for transplantation and/or dialysis access placement.

Somewhat surprisingly, Ricardo et al. found no association between prior nephrology care and clinical outcomes of CKD progression, CVD events or death. However, when the CRIC cohort is carefully considered, these null findings emerge in a different light. The authors aptly point out that certain patient populations with high mortality rates (e.g. advanced liver cirrhosis patients) who, as a consequence of their expected limited survival, are unlikely to be referred to nephrology were excluded from the CRIC study, which might have rendered the non-referred population 'healthier' in ways not accounted for even in the propensity-matched analyses conducted by the authors. They also note the very high achievement of guideline-concordant goals, such as the finding that the majority of CRIC participants were prescribed ACE inhibitors or angiotensin receptor blockers, as a potential contributor to a lack of detectable difference between those who did and did not see a nephrologist. Indeed, the high-quality PCP care as might be expected at academic-affiliated clinics may have led to clinical results akin to those of nephrology care. The potential receipt of care from other specialists who offer management of common CKD complications, including cardiologists and endocrinologists, may have also contributed to the null findings. Still, this report from the CRIC study is a reminder of the potential benefits of collaborative care of CKD and calls for interventions addressing barriers to accessing high-quality primary and nephrology care towards improving CKD outcomes.

Conflicts of Interest: The author declares that he/she does not have a conflict of interest.

Corresponding Author: Deidra C. Crews, MD, ScM; Division of Nephrology, Department of MedicineJohns Hopkins University School of Medicine, Baltimore, MD, USA (e-mail: dcrews1@jhmi.edu).

\section{REFERENCES}

1. Ricardo AC, Roy JA, Tao K, et al. Influence of Nephrologist Care on Management and Outcomes in Adults with Chronic Kidney Disease. J Gen Intern Med. 2015. doi:10.1007/s11606-015-3452-x.

2. Kidney Disease Improving Global Outcomes (KDIGO) Chronic Kidney Disease Working Group. KDIGO 2012 Clinical Practice Guideline for the Evaluation and Management of Chronic Kidney Disease. Kidney Inter. Suppl. 2013;1-150. Kidney Int Suppl (2011). 2012;2(1):3.

3. Saran R, Li Y, Robinson B, et al. US Renal Data System 2014 annual data report: epidemiology of kidney disease in the United States. Am J Kidney Dis. 2015;66(1)(suppl 1):S1-S306.

4. Rao MK, Morris CD, O'Malley JP, Davis MM, Mori M, Anderson S. Documentation and management of CKD in rural primary care. Clin $\mathrm{J} \mathrm{Am}$ Soc Nephrol CJASN. 2013;8(5):739-48.

5. Greer RC, Crews DC, Boulware LE. Challenges perceived by primary care providers to educating patients about chronic kidney disease. J Ren Care. 2012;38(4):174-81.

6. Diamantidis CJ, Powe NR, Jaar BG, Greer RC, Troll MU, Boulware LE. Primary care-specialist collaboration in the care of patients with chronic kidney disease. Clin J Am Soc Nephrol CJASN. 2011;6(2):334-43.

7. Boulware LE, Troll MU, Jaar BG, Myers DI, Powe NR. Identification and referral of patients with progressive CKD: a national study. Am J Kidney Dis. 2006;48(2): 192-204.

8. Agrawal V, Ghosh AK, Barnes MA, McCullough PA. Awareness and knowledge of clinical practice guidelines for CKD among internal medicine residents: a national online survey. Am J Kidney Dis. 2008;52(6):1061-9.

9. Banerjee T, Crews DC, Wesson DE, et al. High Dietary Acid Load Predicts ESRD among Adults with CKD. J Am Soc Nephrol. 2015;26(7):1693-700.

10. Ricardo AC, Anderson CA, Yang W, et al. Healthy lifestyle and risk of kidney disease progression, atherosclerotic events, and death in $\mathrm{CKD}$ : findings from the Chronic Renal Insufficiency Cohort (CRIC) Study. Am J Kidney Dis. 2015;65(3):412-24. 